\title{
The Role of the Amygdala and Olfaction in Unconditioned Fear in Developing Rats
}

\author{
Sean W. C. Chen, ${ }^{1}$ Alexei Shemyakin, ${ }^{2}$ and Christoph P. Wiedenmayer ${ }^{2,3}$ \\ Departments of ${ }^{1}$ Biological Sciences and ${ }^{2}$ Psychiatry, Columbia University, New York, New York 10027, and ${ }^{3}$ Department of Developmental Psychobiology, \\ New York State Psychiatric Institute, New York, New York 10032
}

\begin{abstract}
Early in ontogeny, young rats must be able to detect dangerous stimuli and to exhibit appropriate defensive behaviors. Different nuclei of the amygdala mediate unconditioned and conditioned fear responses to threat in adult rats. The aim of this study was to determine the role of the amygdala in unlearned fear behavior in young rats. When exposed to an unfamiliar adult male rat, preweaning rat pups freeze, with peak levels on postnatal day 14 and declining levels on day 18. Pups were made anosmic to block olfactory input to the amygdala, and amygdala activation was assessed by quantifying the neuronal marker $\mathrm{c}$-fos. Anosmic pups did not freeze in the presence of the male rat and had decreased c-fos expression in the medial amygdala on day 14 and in the medial and lateral amygdala on day 18. However, the decrease in freezing between days 14 and 18 was not associated with a decrease in c-fos expression in the medial amygdala. The medial and lateral amygdala were then inactivated by local muscimol infusion on day 14. Muscimol infusion into the medial amygdala decreased freezing to the male rat but not to a loud noise, whereas infusion into the lateral amygdala blocked freezing to a loud noise but not to the male. These findings indicate that different nuclei of the amygdala process sensory information of different modalities, mediate unconditioned freezing, and may be involved in developmental changes in the fear response in young rats.
\end{abstract}

Key words: amygdala; olfaction; freezing; unconditioned fear; c-fos; development

\section{Introduction}

Research into the neural basis of emotion has identified the amygdala as an important structure in the circuit that underlies the central state of fear. The amygdala receives multimodal sensory input associated with danger and sends processed signals to other brain areas to generate defensive responses (Blanchard and Blanchard, 1972; Kalin et al., 2001; Etkin et al., 2004).

Although the role of the amygdala in detecting and responding to threatening stimuli is recognized, the contribution of each nucleus of the amygdala is still unknown. The lateral and basolateral nuclei are thought to be the primary sites for sensory input and the central nucleus the primary site for output (McDonald, 1998; LeDoux, 2000; Sah et al., 2003; Lanuza et al., 2004). However, recent research has demonstrated that output from the lateral and basolateral amygdala can bypass the central nucleus (Koo et al., 2004).

Another open question concerns the role of the amygdala in the control of learned and unlearned fear. Fear-related defensive behaviors, for example, freezing, can be elicited in adult rats by unconditioned stimuli such as natural predators (Blanchard and Blanchard, 1971). Freezing can also be induced by a previously neutral stimulus that has been paired with an aversive stimulus

Received July 13, 2005; revised 0ct. 16, 2005; accepted 0ct. 24, 2005.

This work was supported by National Institutes of Mental Health Grant MH001975. We thank Harry Shair for technical advice and Stuart Firestein and Michael Myers for helpful comments on this manuscript.

Correspondence should be addressed to Dr. Christoph Wiedenmayer, Department of Psychiatry, Columbia University, 1051 Riverside Drive, Unit 40, New York, NY 10032. E-mail: cpw14@columbia.edu.

DOI:10.1523/JNEUROSCI.2890-05.2006

Copyright $\odot 2006$ Society for Neuroscience $\quad$ 0270-6474/06/260233-08\$15.00/0
(Fanselow and Gale, 2003). Whereas it has been established that the lateral, basolateral, and central nuclei are required for the acquisition and expression of conditioned freezing (Muller et al., 1997; Cousens and Otto, 1998; Nader et al., 2001; Gale et al., 2004), it is still debated which nuclei mediate unconditioned freezing (Vazdarjanova et al., 2001; Wallace and Rosen, 2001; Power and McGaugh, 2002; Choi and Brown, 2003; Fendt et al., 2003; Li et al., 2004).

Little is also known about the role of the amygdala in mediating fear-related behavior during early life, especially with regard to emotional stimuli that are age specific. For instance, an adult unfamiliar, unrelated male rat is an ecologically relevant threat to preweaning but not weaned rats (Paul and Kupferschmidt, 1975; Mennella and Moltz, 1988). In previous work, we found that preweaning pups freeze when exposed to an adult male rat whereas slightly older pups do not freeze to an adult male (Wiedenmayer and Barr, 1998, 2001a). Although the function of this decrease in fear-related behavior is likely related to a change in the threat status of adult males (van Schaik and Janson, 2000), the contribution of the amygdala in mediating these changes is not known.

The aim of this study was to investigate the role played by various nuclei of the amygdala in mediating the unconditioned fear response freezing during early development. Preweaning rat pups were exposed to an adult male rat on postnatal day 14 (P14), when the freezing response peaks, and on P18, when freezing is less pronounced. Pups were made anosmic and the expression of c-fos was used to identify the amygdala nuclei activated by male exposure. These nuclei were then pharmacologically inactivated. 
Finally, the two ages were compared to examine the role of the amygdala in developmental changes in unlearned fear.

\section{Materials and Methods}

Animals. The Long-Evans hooded rats used in this experiment were housed in standard laboratory cages, which were maintained in a colony room with a temperature of $22-24^{\circ} \mathrm{C}$. The animals were given ad libitum access to food and water, and illumination in the room was set to a $12 \mathrm{~h}$ light/dark cycle with light onset at 6:00 A.M. Adult males and females were separated after they had mated. The cages of mated females were checked twice daily at 9:00 A.M. and 5:00 P.M., with rat pups found at either time considered to be $0 \mathrm{~d}$ of age. A sexually experienced, unfamiliar male rat designated as the stimulus animal was housed in the same room. All tests and treatment procedures were approved by and in accordance with the Institutional Animal Care and Use Committee of Columbia University.

Inducing anosmia. We used zinc sulfate $\left(\mathrm{ZnSO}_{4}\right)$ to induce anosmia because the nasal infusion of zinc sulfate selectively destroys the olfactory mucosa and, thus, impairs the function of the main olfactory but not the accessory olfactory system in rat pups (Singh et al., 1976; Hofer and Shair, 1991; Shair et al., 1999). Because zinc sulfate treatment does not seem to have lasting effects, at least in adult rats (Slotnick et al., 2000), the pups were tested within hours (see below) of zinc sulfate treatment. On the morning of the experiment, male rat pups were randomly selected from the litter to be tested, marked with a nontoxic marker (Sharpie; VWR, Bridgeport, NJ), and lightly anesthetized with methoxyflurane (Schering-Plough, Kenilworth, NJ). Each anesthetized pup had a blunted 30 -gauge needle inserted into one nasal cavity, before infusion with either $0.05 \mathrm{ml}$ of a $2.5 \%$ zinc sulfate heptahydrate solution (Sigma-Aldrich, St. Louis, MO) or $0.05 \mathrm{ml}$ of a vehicle solution ( $0.9 \%$ saline). The infusion procedure was judged successful only if the injected solution was seen to come out of the other nostril, and a few drops of blue food coloring (McCormick \& Company, Hunt Valley, MD) were added to both the zinc sulfate and vehicle solutions to make verification easier. Illness behavior, such as hypoactivity and increased mortality, after zinc sulfate infusion has been reported in adult rats (Sieck and Baumbach, 1974; Mayer and Rosenblatt, 1993; Kolunie and Stern, 1995) and have been explained by toxicity of zinc sulfate entering the stomach (Sieck and Baumbach, 1974; Mayer and Rosenblatt, 1977). Therefore, a short perforated plastic tube connected to a syringe was inserted into the esophagus before the infusion procedure to prevent any zinc sulfate solution from entering the pup's stomach (Singh et al., 1976). After infusion, the pups were placed in a holding cage separate from the dam, left on a heating pad, and allowed $4 \mathrm{~h}$ of recovery time before behavioral testing commenced. There were no signs of illness and no death occurred among the zinc sulfate- or saline-infused pups.

Verifying anosmia. Six litters of rat pups not used in any other experiment were used to verify anosmia, with three litters tested per age. Zinc sulfate infusion was performed as described above, and every litter used had two zinc sulfate-treated, two vehicle-treated, and two untreated pups. The dam was anesthetized with a 7:3 ketamine/xylazine mix (both from Phoenix Pharmaceuticals, St. Joseph, MO), with $0.025 \mathrm{ml}$ of the mixture administered per $100 \mathrm{~g}$ of weight. The dam was then placed on her side in an empty testing cage $(26 \times 16 \times 14 \mathrm{~cm})$ containing home cage bedding, and all six pups were introduced into the cage at the same time. The pups were placed individually on the ventrum of the dam in a random order, and given either five (P14) or $10 \mathrm{~min}$ (P18) to attach to a nipple. $\mathrm{P} 18$ rat pups were given an additional $5 \mathrm{~min}$ to attach, because they were closer to being weaned than rat pups on P14. The behavior of the pups was noted visually, with active nosing around the nipple or actual attachment taken as evidence that the pup in question could still smell (Singh et al., 1976). In all six trials, the vehicle-treated and untreated pups (but none of the zinc sulfate-treated pups) attached to a nipple, indicating that zinc sulfate infusion was capable of inducing anosmia.

Surgery. On the afternoon of P13, four male pups from each tested litter were randomly selected for surgery. The pups were anesthetized with methoxyflurane and put in a Kopf stereotaxic apparatus (Kopf Instruments, Tujunga, CA). Bilateral burr holes were then drilled $1.9 \mathrm{~mm}$ posterior and $2.5 \mathrm{~mm}$ lateral to bregma for the medial amygdala, and 2.1 $\mathrm{mm}$ posterior and $4.2 \mathrm{~mm}$ lateral to bregma for the lateral amygdala. Pups then had 26-gauge guide cannulas inserted to a depth of either 7.8 $\mathrm{mm}$ for the medial amygdala or $6.9 \mathrm{~mm}$ for the lateral amygdala. The cannulas were secured to the skull with dental cement. The pups were returned to their litters after they had recovered from the anesthesia. The pups gained weight between surgery and testing the next day (P14), which was taken as evidence of suckling and good recovery.

Testing procedures. Rat pups were tested in groups of three to decrease isolation-induced stress. Each group was placed into one compartment of a testing cage. The testing cage $(46 \times 25 \times 21 \mathrm{~cm})$ used in this experiment was divided using a wire-mesh screen positioned in the middle of the cage, resulting in two equal compartments. The compartment containing the pups had soiled bedding collected from the home cage just before the test. The other compartment was left empty. The pups were allowed to acclimate to their new environment for $5 \mathrm{~min}$, before behavioral testing began.

Pups were tested in male-exposed, control, or noise-exposed experimental conditions. In the male-exposed condition, a sexually experienced, unfamiliar and unrelated adult male rat was placed in the empty compartment for $10 \mathrm{~min}$, and freezing behavior of each pup was recorded by scan sampling every $15 \mathrm{~s}$ by a rater sitting in front of the cage and blind to the treatment. Freezing was defined as any posture in which the pup did not exhibit any movement except that necessary for respiration, and is expressed in the final analysis as a percentage of the scans. In the control condition, the behavior of the rat pups was noted for $10 \mathrm{~min}$ with the other compartment empty. In the noise-exposure condition, a loud noise ( $130 \mathrm{~dB}$ at source) was produced with a personal alarm (Telko, Laguna Hills, CA) held in the other compartment for $15 \mathrm{~s}$ and freezing was recorded for $5 \mathrm{~min}$.

In addition, a set of untreated rat pups on P18 was tested in a special male-exposed experimental condition, where they could smell, but not see, the unfamiliar adult male rat. This experiment was performed by using a piece of cardboard to cover the lower parts of the mesh, thus preventing direct line of sight between the pups and the unfamiliar adult male.

For the anosmia experiments, each testing group consisted of one zinc sulfate-treated, one vehicle-treated and one untreated pup. After completion of testing, the pups were placed in a holding cage containing home cage bedding on a heating pad separate from the dam. Two hours later, the pups were killed, and their brains were collected for immunocytochemistry (see below).

For experiments involving cannulated animals, each testing group consisted of two cannulated and one untreated pup. Cannulated pups were infused with $0.2 \mu \mathrm{l}$ of a $2 \mathrm{mg} / \mathrm{ml} \mathrm{GABA}$ agonist, muscimol, (SigmaAldrich), or $0.2 \mu \mathrm{l}$ of a vehicle solution ( $0.9 \%$ saline). The 30 -gauge injection cannula was connected to a $10 \mu$ l glass syringe (Hamilton, Reno, NV), operated by hand, and was left in the guide cannula for $30 \mathrm{~s}$ after the injection to permit maximal diffusion. Injection cannulas for both ages were constructed so that they extended $0.5 \mathrm{~mm}$ beyond the tip of the guide cannula. All cannulated pups were subsequently killed, and their brains collected for verification of cannula placement.

Radiolabeling. To assess the extent of muscimol spread within the amygdala, we infused tritium $\left[{ }^{3} \mathrm{H}\right]$-labeled muscimol at the same dosage and volume as above $(0.4 \mu \mathrm{g}$ in $0.2 \mu \mathrm{l} ; 0.2 \mu \mathrm{Ci})$. Five minutes after infusion, the rat pups were decapitated and had their brains removed. The brains were frozen on dry ice, sectioned at $30 \mu \mathrm{m}$ in a cryostat, dried by heat dessication and apposed to film (BioMax MR; Sigma-Aldrich) for $14 \mathrm{~d}$. The resulting autoradiograms were analyzed by superimposing them over cresyl violet-stained sections that were adjacent to the sections on the film.

Immunocytochemistry. Two hours after testing, when Fos expression levels were expected to be at a peak (Morgan and Curran, 1991), the rat pups were removed from the holding cage and given an overdose volume of sodium pentobarbital (Abbott Laboratories, North Chicago, IL). Transcardial perfusion of the pups was performed using a $10 \%$ formalin solution (Sigma-Aldrich) as the fixative, and the brains were removed and stored in formalin overnight. The brains were subsequently put in a $30 \%$ sucrose buffer for cryoprotection before they were frozen and sec- 
tioned in a cryostat. Each section was $30 \mu \mathrm{m}$ thick, and six wells of three sections each were collected from the amygdala. Seven sections were skipped between each well. From each well, one section was placed on a microscope slide for cresyl violet staining to locate the brain areas of interest, and two slices were processed immunocytochemically (one as a backup). Sections of male-exposed and control animals were assayed together.

The brain sections were processed using a modified protocol provided by a commercial antibody staining kit (ABC kit; Vectastain Elite; Vector Laboratories, Burlingame, CA), which uses the diaminobenzidineperoxidase method of visualizing antigen-binding sites. The sections were first incubated for $48 \mathrm{~h}$ at $4^{\circ} \mathrm{C}$ in the primary antibody, rabbit anti-Fos (c-fos; H-125; Santa Cruz Biotechnology, Santa Cruz, CA), diluted 1:2000 in PBS with Triton X-100 and 1\% normal goat serum. The sections were then rinsed and incubated with the secondary antibody (goat anti-rabbit; Vector Laboratories) for $1 \mathrm{~h}$, and processed using the $\mathrm{ABC}$ kit protocol. Stained sections were mounted on gelatin-covered slides, dehydrated in alcohol and xylene, and coverslipped.

Fos data acquisition and analysis. Positive-labeled Fos-like immunoreactive cells were visualized using a microscope [Leitz (Wetzlar, Germany), with a $50 \times$ objective] equipped with a drawing tube that provided sufficient magnification to identify all Fos-positive cells. Brain nuclei of the amygdaloid complex were outlined with the cresyl violetstained sections using an atlas of the rat brain (Paxinos and Watson, 1998), before the Fos-stained sections were superimposed over them. All Fos-positive cells were counted bilaterally in the relevant brain nuclei by a person unaware of the treatment conditions. For a cell to be considered Fos positive, it had to be distinct from the background, regardless of the intensity of the staining. Sections of male-exposed and control animals were matched for corresponding neuroanatomical levels and the mean number of cell counts per brain area was calculated by averaging the counts from all sections of each animal.

Histology. Dissected brains were placed in formalin overnight before they were transferred to a $30 \%$ sucrose solution for cryoprotection. Fixed and frozen brains were sectioned coronally at $30 \mu \mathrm{m}$ through the amygdala, mounted on glass slides, stained with cresyl violet, and coverslipped. Cannula placement was determined for each rat using a brain atlas (Paxinos and Watson, 1998).

Statistical analysis. All data were analyzed by factorial ANOVA, followed by Newman-Keuls tests for post hoc comparisons. Statistical analyses of test conditions were done at a within-group level, whereas comparisons of the two age groups were done at a between-group level.

\section{Results}

Freezing to aversive stimuli on P14

The freezing behavior of $\mathrm{P} 14$ rat pups ( $n=7$, where $n=$ number of litters) exposed to an unfamiliar adult male or an empty cage differed across test conditions (ANOVA; $F_{(5,30)}=41.0 ; p<$ 0.001). Untreated and vehicle-treated pups exposed to the male froze significantly more than untreated and vehicle-treated pups in the control test condition (Newman-Keuls; $p<0.001$ ). In contrast, zinc sulfate-treated pups froze significantly less than untreated and vehicle-treated pups when they were exposed to the male $(p<0.001)$. There was no significant difference in freezing behavior between zinc sulfate-treated pups exposed to the male and zinc sulfate-treated control pups (Fig. 1). To ensure that the infusion procedure had not interfered with the pup's ability to freeze, a separate set of $\mathrm{P} 14$ pups $(n=7)$ was exposed to a loud noise. There were no significant differences in the levels of freezing across untreated, vehicle-treated, and zinc sulfatetreated pups (Fig. 1).

\section{Fos expression in the amygdala on $\mathrm{P} 14$}

Fos expression was quantified in four nuclei (lateral, basolateral, medial, and central) of the amygdala in vehicle and zinc sulfatetreated rat pups $(n=7)$ that were control or male-exposed. A main effect for test conditions was found for the medial amygdala

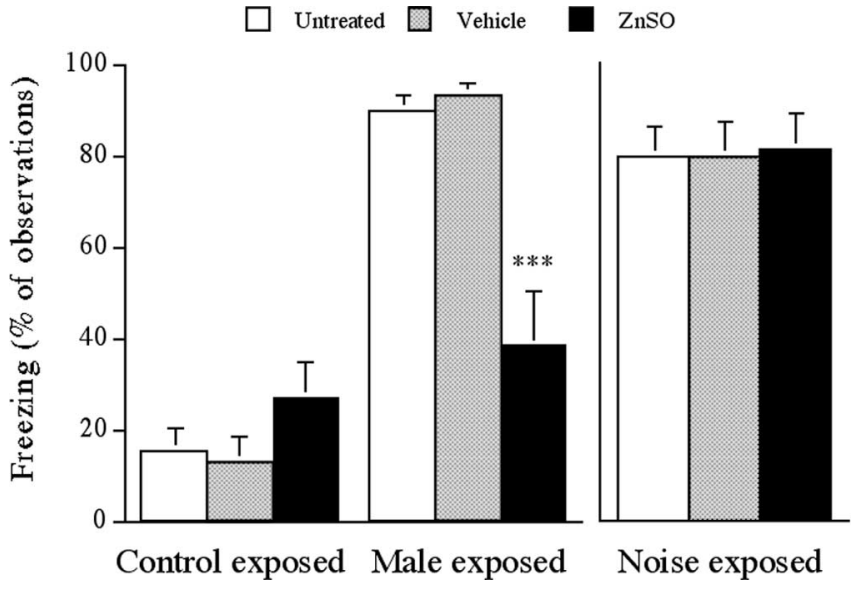

Figure 1. Threat induced freezing in P14 rats. Rats were exposed to an empty cage (control exposed), an unfamiliar adult male rat (male exposed), or a loud noise (noise exposed; $n=7$; mean $\pm \mathrm{SE}$ ). Vehicle, Vehicle treated; ZnSO, zinc sulfate treated. ${ }^{* * *} p<0.001$, significantly different from untreated and vehicle-treated exposed to the male.

$\left(F_{(3,18)}=14.8 ; p<0.001\right)$ but not for the lateral, basolateral, or central amygdala. In vehicle-treated animals, male exposure significantly increased the number of Fos-positive cells in the medial amygdala compared with control exposure $(p<0.01)$. In contrast, zinc sulfate-treated pups exposed to the male had significantly reduced Fos expression in the medial amygdala compared with vehicle-treated pups exposed to the male $(p<0.001)$. There was no significant difference in the number of Fos-positive cells when zinc sulfate male-exposed and zinc sulfate control-exposed pups were compared (Fig. $2 A$ ). Examples of the distribution of Fospositive cells in the amygdala on P14 are displayed in Figure $2 B$.

Freezing behavior after medial amygdala inactivation on P14 Because male exposure activated only the medial amygdala, we inactivated this nucleus with a local infusion of muscimol and subsequently exposed pups to an unfamiliar adult male. All pups used in this experiment had cannula tips located within the medial amygdala (Fig. 3A), with pups from four litters excluded because the cannula tips were located outside the medial amygdala. We also assessed muscimol spread by using tritium $\left({ }^{3} \mathrm{H}\right)$ labeled muscimol and found that a radioactive signal was detectable at a maximum radius of $0.5 \mathrm{~mm}$ from the cannula tip in the medial amygdala (Fig. 4).

The freezing behavior of P14 rat pups $(n=8)$ differed significantly across test conditions $\left(F_{(5,35)}=37.5 ; p<0.001\right)$. Untreated and vehicle-treated rat pups froze significantly more when exposed to the male, compared with the untreated and vehicle-treated pups in the control test condition $(p<0.001)$. In contrast, muscimol-treated pups exposed to the male froze significantly less when compared with untreated and vehicle-treated pups exposed to the male $(p<0.001)$. There were no significant differences in freezing when the behavior of muscimol-treated pups exposed to the male was compared with the behavior of untreated and vehicle-treated pups in the control condition (Fig. 3B).

Because inactivation of the medial amygdala could affect the ability or motivation of rat pups to freeze, pups $(n=6)$ not used in any other experiment were exposed to an aversive stimulus (a loud noise) of a different sensory modality. There were no differences in freezing behavior, when untreated, vehicle-treated, and muscimol-treated pups were exposed to a loud noise (Fig. 3B). 
Freezing behavior after lateral amygdala inactivation on $\mathrm{P} 14$

To determine whether the medial amygdala selectively mediates male-induced freezing, the lateral amygdala was inactivated. All rat pups used in this experiment had cannula tips located within the lateral amygdala (Fig. 3C), with pups from three litters excluded because the cannula tips were located outside the lateral amygdala.

The freezing behavior of P14 rat pups cannulated in the lateral amygdala $(n=7)$ differed significantly across test conditions $\left(F_{(5,30)}=34.6 ; p<0.001\right)$, with untreated, vehicle-treated, and muscimol-treated pups exposed to the male freezing significantly more than untreated, vehicle-treated, and muscimol infused pups in the control test condition $(p<0.001)$. There were no significant differences in freezing when untreated, vehicle-treated and muscimoltreated animals in the male or control test conditions were compared (Fig. 3D).

When pups cannulated in the lateral amygdala $(n=7)$ were exposed to a loud noise, freezing behavior differed across tests conditions $\left(F_{(2,12)}=5.1 ; p<0.05\right)$. Muscimol-treated pups froze significantly less compared with untreated and vehicletreated rats when they were exposed to a loud noise $(p<0.05)$, whereas the freezing behavior of untreated and vehicletreated pups did not differ (Fig. 3D).

\section{Freezing to aversive stimuli on P18}

Next, we examined freezing behavior in response to the adult male rat in slightly older rat pups. Freezing behavior differed significantly across testing conditions when P18 rats were exposed to the adult male rat compared with controls $\left(F_{(5,30)}=\right.$ $14.1 ; p<0.001)$, with untreated and vehicle-treated rat pups exposed to the male freezing significantly more than untreated and vehicle-treated pups from the control condition $(p<0.001)$. Within the male-exposed group, zinc sulfate-treated pups froze significantly less than untreated and vehicle-treated pups $(p<0.001)$. The freezing behavior of zinc sulfate-treated pups exposed to the male did not differ from pups in the control test condition (Fig. 5). The freezing levels of P18 pups $(n=6)$ exposed to a loud noise also did not differ between the untreated, vehicle-treated, or zinc sulfate-treated animals (Fig. 5).

Because rat pups open their eyes around postnatal day 15 (Alberts, 1984), we investigated whether freezing on P18 depended on visual cues. P18 pups $(n=8)$ were exposed to the adult male rat placed behind an opaque screen. The freezing levels of pups exposed to a male behind an opaque screen or the wire mesh screen were compared (data not shown). There was a main effect for test

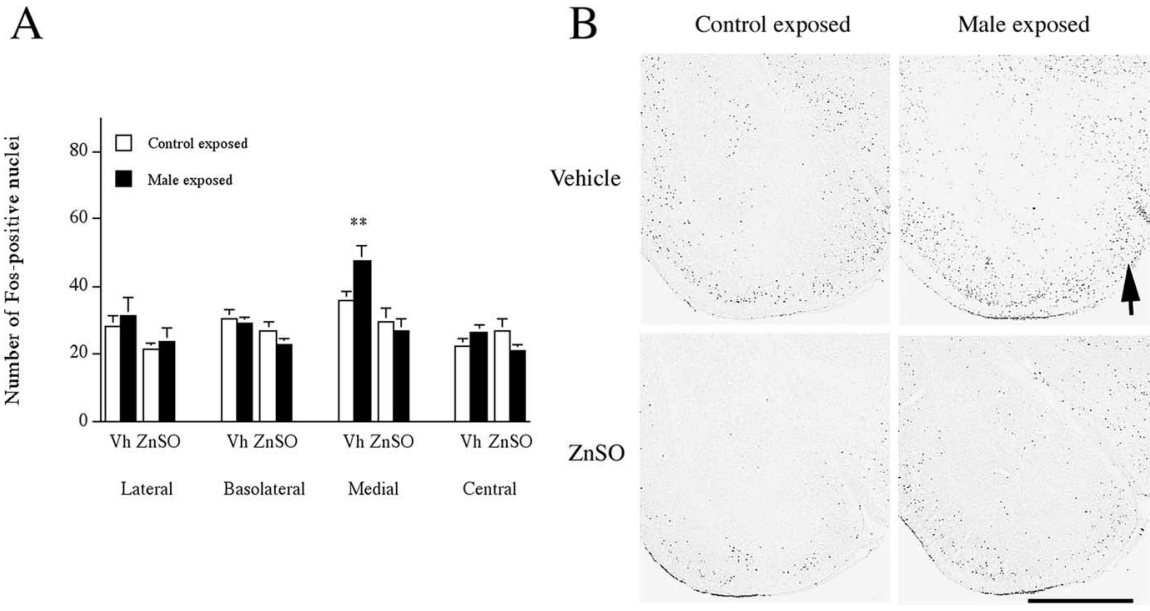

Figure 2. Anosmia prevents male-induced c-fos expression in medial amygdala of P14 rats. $A$, Number of Fos-positive nuclei in nuclei of the amygdala of vehicle-treated and zinc sulfate-treated pups exposed to the male or control exposed $(n=7$; mean \pm SE). Vh, Vehicle treated; ZnSO, zinc sulfate treated. ${ }^{* *} p<0.01$, significantly different from vehicle-treated control exposed. $\boldsymbol{B}$, Photomicrographs of Fos expression in the amygdala. Arrow indicates medial amygdala. For anatomical boundaries of the nuclei, see Figure $3 A$. Scale bar, $1 \mathrm{~mm}$.

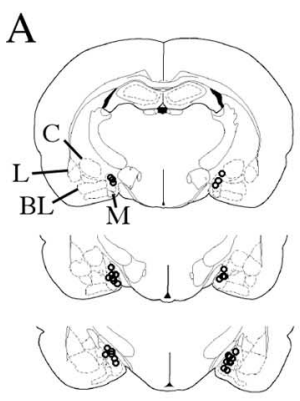

Vehicle infused

C

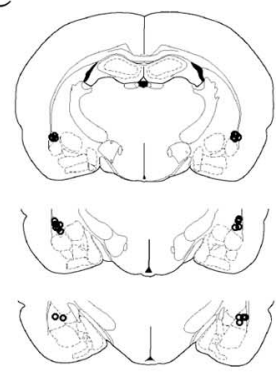

Vehicle infused

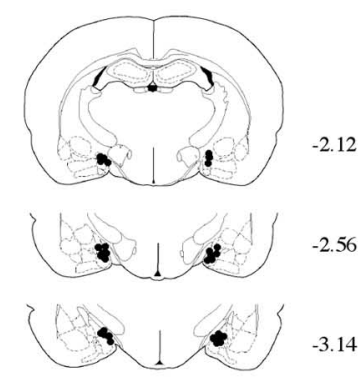

Muscimol infused
B

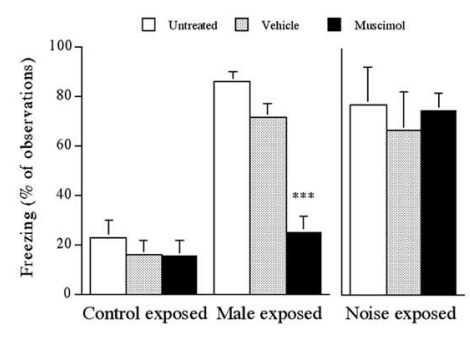

D
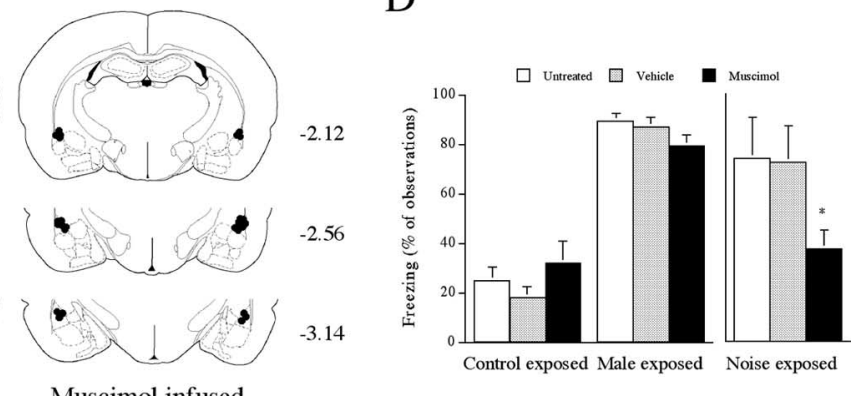

Figure 3. Inactivation of nuclei of the amygdala reduces threat-induced freezing in P14 rats. $\boldsymbol{A}$, Cannula tip placement for animals of the medial amygdala group. Open circles represent the location of cannula tips in rats infused with vehicle, and closed circles represent the location of cannula tips in rats infused with muscimol. Numbers indicate the distance in millimeters from the bregma. L, Lateral nucleus; BL, basolateral nucleus; $C$, central nucleus; $M$, medial nucleus. $B$, Freezing (mean \pm SE) of rats cannulated in the medial amygdala exposed to an empty cage, an unfamiliar adult male rat $(n=8)$, or a loud noise $(n=6)$. ${ }^{* * *} p<0.001$, significantly different from untreated and vehicle-treated exposed to the male. $\boldsymbol{C}$, Cannula tip placement for animals of the lateral amygdala group. Open circles represent the location of cannula tips in rats infused with vehicle, and closed circles represent the location of cannula tips in rats infused with muscimol. $\boldsymbol{D}$, Freezing of rats cannulated in the lateral amygdala exposed to an empty cage, an unfamiliar adult male rat, or a loud noise (mean $\pm \mathrm{SE} ; n=7$ ). ${ }^{*} p<0.05$, significantly different from untreated and vehicle-treated exposed to noise.

condition $\left(F_{(1,13)}=57.4 ; p<0.001\right)$ but not for screen type, and no interaction was found for testing condition and screen type.

\section{Fos expression in the amygdala on P18}

Fos expression on P18 was quantified in the four nuclei of the amygdala of vehicle- and zinc sulfate-treated rat pups $(n=7)$ 


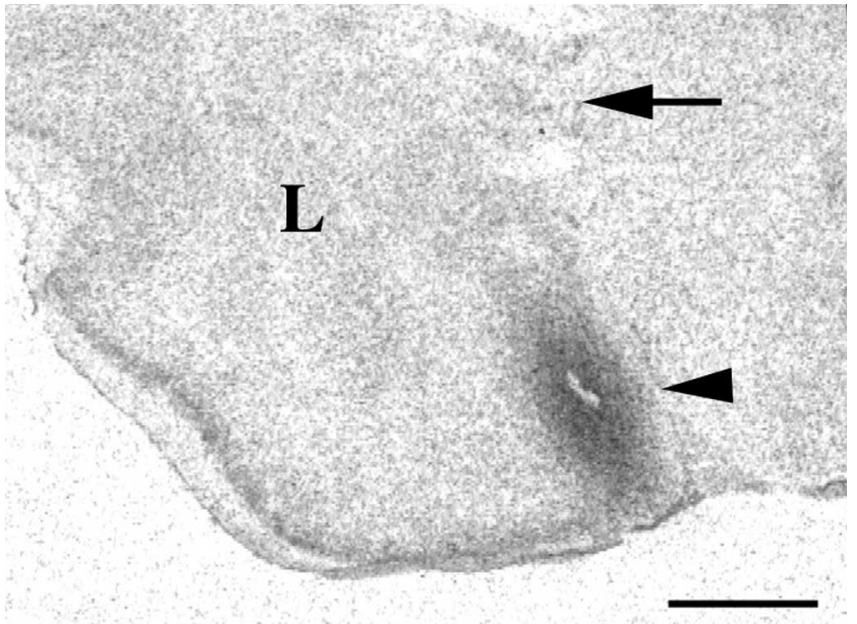

Figure 4. Visualization of muscimol diffusion from cannula tip $5 \mathrm{~min}$ after infusion into the medial amygdala on P14. Autoradiogram of ${ }^{3} \mathrm{H}$-labeled muscimol is superimposed over a cresyl violet-stained section. Scale bar, $1 \mathrm{~mm}$. Arrow, Cannula tract; arrowhead, cannula tip; L, lateral amygdala.

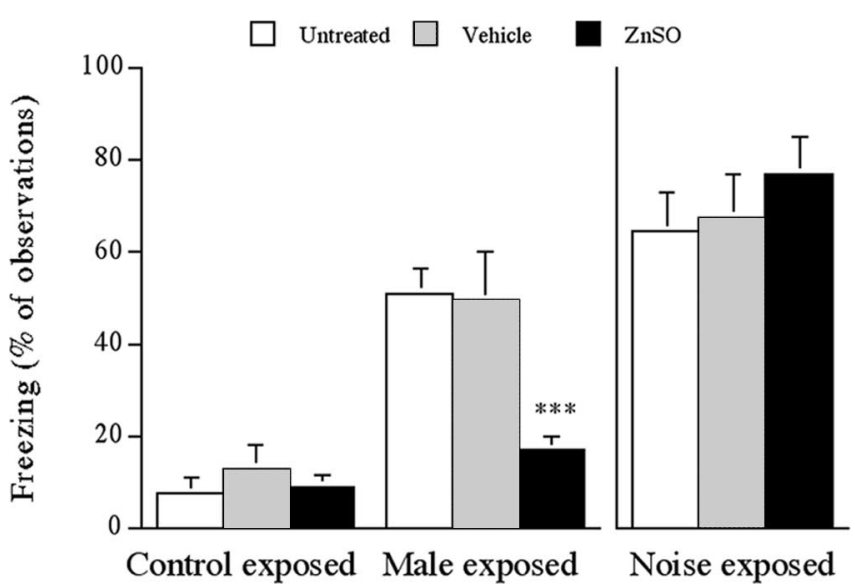

Figure 5. Threat induced freezing in P18 rats. Rats were exposed to an empty cage, an unfamiliar adult male rat $(n=7)$, or a loud noise $(n=6$; mean \pm SE). Vehicle, Vehicle treated; $\mathrm{ZnSO}$, zinc sulfate treated. ${ }^{* * *} p<0.001$, significantly different from untreated and vehicletreated exposed to the male.

exposed to the male or the control. A main effect for test conditions was found for the medial amygdala $\left(F_{(3,18)}=20.5 ; p<\right.$ $0.001)$. In vehicle-treated pups, male exposure significantly increased the number of Fos-positive cells in the medial amygdala, compared with pups in the control test condition $(p<0.001)$. In contrast, zinc sulfate-treated pups exposed to the adult male rat had significantly reduced number of cells in the medial amygdala, compared with vehicle-treated pups exposed to the same male $(p<0.001)$. No significant differences in Fos levels in the medial amygdala were found when zinc sulfate-treated pups exposed to the unfamiliar male were compared with control pups (Fig. 6A).

Unlike P14 pups, significant differences in the number of Fospositive cells on P18 were also detected in the lateral amygdala $\left(F_{(3,18)}=5.7 ; p<0.01\right)$. Vehicle-treated pups exposed to the male had significantly more Fos-positive cells than vehicle-treated control animals $(p<0.01)$. In contrast, the zinc sulfate-treated rats exposed to the male did not differ from controls and also had significantly less Fos-positive cells than the vehicle-treated pups exposed to the male $(p<0.01)$ (Fig. $6 A$ ). The distribution of Fos-positive cells in the amygdala on P18 is displayed in Figure $6 B$.

\section{Differences between P14 and P18}

During development, a stimulus may lose its property to induce a defensive response. Rat pups display high levels of freezing to an adult male on P14 but no longer freeze in response to a male on postnatal day 26 (Wiedenmayer and Barr, 1998, 2001a). To determine the developmental changes in fear-related behavior, we compared freezing levels in response to the male and a loud noise on P14 and P18 in untreated and vehicle-treated animals (Figs. 1, 5).

There were main effects for age (untreated, $F_{(1,12)}=29.7, p<$ 0.001 ; vehicle, $\left.F_{(1,12)}=12.4, p<0.01\right)$ and exposure condition (untreated, $F_{(1,12)}=186.3, p<0.001$; vehicle, $F_{(1,12)}=77.2, p<$ 0.001 ), and a significant interaction (untreated, $F_{(1,12)}=14.0$, $p<0.01$; vehicle, $\left.F_{(1,12)}=10.7, p<0.01\right)$. Male-exposed rats froze significantly less on day 18 than on day $14(p<0.001)$. However, freezing levels to a loud noise did not differ between P14 and P18 in untreated and vehicle-treated pups.

To examine this age-dependent decrease in freezing in the context of amygdala activation, the number of Fos-positive cells in the medial amygdala was compared between the two age groups in vehicle-treated rats (Figs. $2 A, 6 A$ ). Although there were main effects for age $\left(F_{(1,12)}=23.8 ; p<0.001\right)$ and exposure condition $\left(F_{(1,12)}=21.8 ; p<0.001\right)$, no interaction between these two variables was found. This indicates that although the number of Fos-positive cells was higher in both control and maleexposed animals on P18 as compared with P14, the difference between controls and male exposed animals was similar at the two ages. In other words, decreased freezing in response to a male rat on P18 was not associated with decreased Fos expression in the medial amygdala.

\section{Discussion}

Our data demonstrate that different nuclei of the amygdala play distinct roles in mediating an unconditioned fear response to threat in young rats; the medial amygdala mediates freezing to olfactory cues from a potentially infanticidal adult male rat, whereas the lateral amygdala mediates freezing to a loud noise. However, although freezing to a loud noise did not change between P14 and P18, freezing to the male decreased in P18 pups, demonstrating that unconditioned fear responses can undergo age-related and stimulus-specific changes.

Anosmic pups did not freeze in the presence of the adult male rat, indicating that pups use odor cues to detect this potential threat. Because adult rats also freeze when exposed to odors of various predator species (Wallace and Rosen, 2000; Vazdarjanova et al., 2001; de Paula et al., 2005), our findings suggest that this reliance on olfactory cues to sense danger extends throughout a rat's life. Olfactory information is conveyed from the olfactory bulb through dense, direct connections to the medial amygdala and through indirect, light projections to the lateral and central amygdala (Scalia and Winans, 1975; McDonald, 1998; Sah et al., 2003). Male exposure increased c-fos expression in vehicle-treated but not in zinc sulfate-treated pups in the medial amygdala on P14 and in the medial and lateral amygdala on P18. Although the medial amygdala has been implicated in the detection of pheromones via the accessory olfactory system (Meredith, 1998; Dielenberg and McGregor, 2001), the involvement of the accessory olfactory system in the processing of male odor seems unlikely because zinc sulfate selectively damages the main olfactory system in rat pups (Singh et al., 1976). However, because the main olfactory system can process pheromones as well (Johnston, 1998), it cannot be excluded that chemosignals emitted by the male include pheromones.

Exposure to predator odors has recently been used to investi- 
gate the neural basis of unlearned fear in adult rats (Canteras, 2002; Rosen, 2004). Exposure to cat odor induced c-fos expression in the posterior subdivision of the medial amygdala but not in the lateral, basolateral, or central amygdala (Dielenberg et al., 2001; Figueiredo et al., 2003; McGregor et al., 2004). We did not distinguish between anterior and posterior subdivisions of the medial amygdala and further investigation is needed to determine whether exposure to odor of a dangerous conspecific, the male rat, activates the same amygdala subdivisions in young rats as exposure to heterospecific predators does in adult rats.

In a previous study, male exposure induced c-fos expression in the lateral amygdala on P14 (Wiedenmayer and Barr, 2001b). This discrepancy could be explained by differences in the experimental protocols used, because pups in the present study were kept in a holding cage separate from the dam after exposure, whereas pups from our previous study were returned to their home cage immediately after exposure. The presence of conspecifics can diminish the consequences of an aversive experience. c-fos expression patterns in limbic areas including the amygdala were altered in adult rats when they were housed in groups instead of individually during chronic stress exposure (Westenbroek et al., 2003). Accordingly, the presence of the dam could have affected subsequent patterns of c-fos expression in the pups. The mechanisms of how social variables alter an individual's experience of fear merit further investigation.

Although Fos expression indicates that the amygdala plays a role in the detection of aversive stimuli, it does not necessarily indicate that the amygdala is involved in mediating the resultant behavioral response (Holahan and White, 2004). Therefore, to demonstrate a direct relationship between amygdala activation and freezing, we temporarily inactivated the medial and lateral amygdala in 14-d-old rats with muscimol, a GABA agonist (Andrews and Johnston, 1979), and with tritiated muscimol investigated how far the drug spread. In agreement with similar studies using autoradiography in adult rats (Martin, 1991; Edeline et al., 1999), we found that the spread of muscimol was limited to the infused nucleus. We also found that inactivation of the medial amygdala suppressed freezing to the adult male, but not to a loud noise. Therefore, inactivating the medial amygdala did not interfere with the pups' ability to execute the freezing response, but rather, the pups' ability to perceive the adult male as a threat. In contrast, lateral amygdala inactivation had no effect on maleinduced freezing but suppressed freezing to a loud noise. These findings strongly suggest that the medial and lateral amygdala process aversive stimuli of different sensory modalities, whereas other structures may generate the actual freezing response. One such area that controls defensive behavior and receives amygdala projections is the periaqueductal gray in the midbrain (Bandler and Shipley, 1994). We have demonstrated previously that maleinduced levels of c-fos expression in the periaqueductal gray correlate with freezing and that periaqueductal gray lesion decreased levels of freezing on P14 (Wiedenmayer et al., 2000; Wiedenmayer and Barr, 2001b).

The majority of studies that have examined the role of differ-

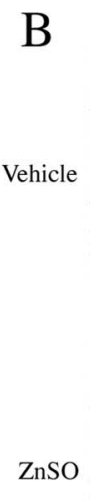

Control exposed

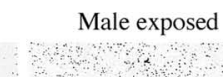

Figure 6. Anosmia prevents male-induced c-fos expression in lateral and medial amygdala of P18 rats. $\boldsymbol{A}$, Number of Fospositive nuclei in nuclei of the amygdala of vehicle-treated and zinc sulfate-treated pups exposed to the male- or control-exposed from vehicle-treated control exposed. $\boldsymbol{B}$, Photomicrographs of Fos expression in the amygdala. Arrows indicate the lateral and boundaries of the nuclei, see Figure $3 A$. Scale bar, $1 \mathrm{~mm}$

ent nuclei of the amygdala in unconditioned fear-related behavior have exposed adult rats to predator cues. Electrolytic lesion encompassing all nuclei of the amygdala decreased freezing to a cat (Blanchard and Blanchard, 1972). Neurotoxic lesions of the lateral amygdala that spared fibers of passage did not affect freezing to trimethylthiazoline (TMT), a component of fox feces (Wallace and Rosen, 2001). In a similar study, muscimol inactivation of the lateral and central amygdala also did not affect TMT induced freezing (Fendt et al., 2003). However, the exact role of the lateral and basolateral amygdala in unconditioned freezing remains controversial, because another study has shown that both temporary inactivation and neurotoxic lesions of the lateral and basolateral amygdala suppressed freezing to a ball of cat hair (Vazdarjanova et al., 2001). In contrast, the medial amygdala appears to be necessary in odor-induced unconditioned freezing because neurotoxic lesion diminished freezing in response to cat odor (Li et al., 2004). Therefore, convergent findings indicate that the medial amygdala mediates the expression of unconditioned fear responses to olfactory stimuli in both young and adult rats.

In the present study, although the freezing levels in response to an adult male rat changed between P14 and P18, there were no changes in freezing levels with regard to a loud noise. The decrease in unconditioned freezing to an adult male rat parallels changes in infanticidal threat imposed by the male (Wiedenmayer and Barr, 1998) and are in opposite direction to freezing to cat odor, which signals threat throughout development (Hubbard et al., 2004; Wiedenmayer et al., 2005). Young rat thus possess an innate competence to respond appropriately to the levels of actual threat. The age-dependent decrease in freezing could indicate a decreased state of fear and could be mediated by changes in the neural substrate underlying this behavior.

Visual detection of the male by pups on P18 may have modulated olfactory input into the amygdala and resulted in decreased freezing. Visual input could also explain the increase in lateral amygdala c-fos expression on P18, because visual information is processed in the lateral nucleus (McDonald, 1998). However, anosmic pups on P18 had decreased c-fos expression in the lateral amygdala, and pups that were exposed to the adult male behind an opaque screen still froze, indicating that visual cues 
from the male are unlikely to play a role in modulating freezing at that age.

Structures, such as the prefrontal cortex, that send direct inhibitory projections to the amygdala (Paré et al., 2004) could modulate freezing by exerting inhibitory control on subcortical and midbrain regions. However, a comparison of c-fos levels in the medial amygdala between P14 and P18 argues against a direct inhibition of this nucleus by another structure because the relative number of male-induced Fos-positive cells was the same at both ages. Alternatively, inhibitory structures may exert their effects on areas involved in freezing downstream of the amygdala, for example at the level of the periaqueductal gray (Keay and Bandler, 2001).

Finally, maturational processes within the amygdala itself could contribute to the changes in male-induced freezing. The increased number of Fos-positive cells on P18 could indicate changes in synaptic connections within or between nuclei. Dense projections interconnect amygdala nuclei (Pitkänen, 2000) and form intrinsic inhibitory and excitatory networks (Paré et al., 2003). It is possible that amygdala nuclei could process cues from the adult male differently depending on age, resulting in altered output function and, hence, freezing levels. Male induced activation of the lateral amygdala on P18 but not P14 supports this hypothesis. It is also possible that changes in the central amygdala underlie the changing levels of freezing, because the central nucleus can modulate fear and stress responses by gating synaptic output (Kang-Park et al., 2004; Day et al., 2005; Huber et al., 2005). However, its role in decreasing male-induced freezing in young rats is questionable, because central amygdala lesions do not reduce freezing to cat odor in adult rats (Li et al., 2004).

In summary, our findings suggest that young rats have the ability to exhibit unlearned fear-related behavior in response to threatening stimuli and that this competence is mediated by specific nuclei of the amygdala.

\section{References}

Alberts JR (1984) Sensory-perceptual development in the Norway rat: a view toward comparative studies. In: Comparative perspectives on the development of memory (Kail R, Spear NE, eds), pp 65-101. Hillsdale, NJ: Lawrence Erlbaum Associates.

Andrews PR, Johnston GAR (1979) GABA agonists and antagonists. Biochem Pharmacol 28:2697-2702.

Bandler R, Shipley MT (1994) Columnar organization in the midbrain periaqueductal gray: modules for emotional expression? Trends Neurosci 17:379-389.

Blanchard DC, Blanchard RJ (1972) Innate and conditioned reactions to threat in rats with amygdaloid lesions. J Comp Physiol Psychol 81:281-290.

Blanchard RJ, Blanchard DC (1971) Defensive reactions in the albino rat. Learn Motiv 2:352-362.

Canteras NS (2002) The medial hypothalamic defensive system: hodological organization and functional implications. Pharmacol Biochem Behav 71:481-491.

Choi J-S, Brown TH (2003) Central amygdala lesions block ultrasonic vocalization and freezing as conditional but not unconditional responses. J Neurosci 23:8713-8721.

Cousens G, Otto T (1998) Both pre- and posttraining excitotoxic lesions of the basolateral amygdala abolish the expression of olfactory and contextual fear conditioning. Behav Neurosci 112:1092-1103.

Day HEW, Nebel S, Sasse S, Campeau S (2005) Inhibition of the central extended amygdala by loud noise and restraint stress. Eur J Neurosci 21:441-454.

de Paula HMG, Gouveira A, de Almeida MV, Hoshino K (2005) Anxiety levels and wild running susceptibility in rats: assessment with elevated plus maze test and predator odor exposure. Behav Proc 68:135-144.

Dielenberg RA, McGregor IS (2001) Defensive behavior in rats towards predatory odors: a review. Neurosci Biobehav Rev 25:597-609.
Dielenberg RA, Hunt GE, McGregor IS (2001) "When a rat smells a cat": the distribution of Fos immunoreactivity in rat brain following exposure to a predatory odor. Neuroscience 104:1085-1097.

Edeline J-M, Hars B, Hennevin E, Cotillon N (1999) Muscimol diffusion after intracerebral microinjections: a reevaluation based on electrophysiological and autoradiographic quantifications. Neurobiol Learn Mem 78:100-124.

Etkin A, Klemenhagen KC, Dudman JT, Rogan MT, Hen R, Kandel ER, Hirsch J (2004) Individual differences in trait anxiety predict the response of the basolateral amygdala to unconsciously processed fearful faces. Neuron 44:1043-1055.

Fanselow MS, Gale GD (2003) The amygdala, fear, and memory. Ann NY Acad Sci 985:125-134.

Fendt M, Endres T, Apfelbach R (2003) Temporary inactivation of the bed nucleus of the stria terminalis but not of the amygdala blocks freezing induced by trimethylthiazoline, a component of fox feces. J Neurosci 23:23-28.

Figueiredo HF, Bodie BL, Tauchi M, Dolgas CM, Herman JP (2003) Stress integration after acute and chronic predator stress: differential activation of central stress circuitry and sensitization of the hypothalamo-pituitaryadrenocortical axis. Endocrinol 144:5249-5258.

Gale GD, Anagnostaras SG, Godsil BP, Mitchell S, Nozawa T, Sage JR, Wiltgen B, Fanselow MS (2004) Role of the basolateral amygdala in the storage of fear memories across the adult lifetime of rats. J Neurosci 24:3810-3815.

Hofer MA, Shair HN (1991) Trigeminal and olfactory pathways mediating isolation distress and companion comfort responses in rat pups. Behav Neurosci 105:699-706.

Holahan MR, White NM (2004) Amygdala c-Fos induction corresponds to unconditioned and conditioned aversive stimuli but not to freezing. Behav Brain Res 152:109-120.

Hubbard DT, Blanchard DC, Yang M, Markham CM, Gervacio A, Chun-I. L, Blanchard RJ (2004) Development of defensive behavior and conditioning to cat odor in the rat. Physiol Behav 80:525-530.

Huber D, Veinante P, Stoop R (2005) Vasopressin and oxytocin excite distinct neuronal populations in the central amygdala. Science 308:245-248.

Johnston RE (1998) Pheromones, the vomeronasal system, and communication. Ann NY Acad Sci 855:333-348.

Kalin NH, Shelton SE, Davidson RJ, Kelley AE (2001) The primate amygdala mediates acute fear but not the behavioral and physiological components of anxious temperament. J Neurosci 21:2067-2074.

Kang-Park M-H, Wilson WA, Moore SD (2004) Differential actions of diazepam and zolpidem in basolateral and central amygdala nuclei. Neuropharmacol 46:1-9.

Keay KA, Bandler R (2001) Parallel circuits mediating distinct emotional coping reactions to different types of stress. Neurosci Biobehav Rev 25:669-678.

Kolunie JM, Stern JM (1995) Maternal aggression in rats: effects of olfactory bulbectomy, $\mathrm{ZnSO}_{4}$-induced anosmia, and vomeronasal organ removal. Horm Behav 29:492-518.

Koo JW, Han J-S, Kim JJ (2004) Selective neurotoxic lesions of basolateral and central nuclei of the amygdala produce differential effects on fear conditioning. J Neurosci 24:7654-7662.

Lanuza E, Nader K, LeDoux JE (2004) Unconditioned stimulus pathways to the amygdala: effects of posterior thalamic and cortical lesions on fear conditioning. Neuroscience 125:305-315.

LeDoux JE (2000) Emotion circuits in the brain. Annu Rev Neurosci 23:155-184.

Li C-I, Maglinao TL, Takahashi LK (2004) Medial amygdala modulation of predator odor-induced unconditioned fear in the rat. Behav Neurosci 118:324-332.

Martin JH (1991) Autoradiographic estimation of the extent of reversible inactivation produced by microinjection of lidocaine and muscimol in the rat. Neurosci Lett 127:160-164.

Mayer AD, Rosenblatt JS (1977) Effects of intranasal zinc sulfate on open field and maternal behavior in female rats. Physiol Behav 18:101-109.

Mayer AD, Rosenblatt JS (1993) Peripheral olfactory deafferentation of the primary olfactory system in rats using $\mathrm{ZnSO}_{4}$ nasal spray with special reference to maternal behavior. Physiol Behav 53:587-592.

McDonald AJ (1998) Cortical pathways to the mammalian amygdala. Prog Neurobiol 55:257-332.

McGregor IS, Hargreaves GA, Apfelbach R, Hunt GE (2004) Neural corre- 
lates of cat odor-induced anxiety in rats: region-specific effects of the benzodiazepine midazolam. J Neurosci 24:4134-4144.

Mennella JA, Moltz H (1988) Infanticide in rats: male strategy and female counter-strategy. Physiol Behav 42:19-28.

Meredith M (1998) Vomeronasal, olfactory, hormonal convergence in the brain. Ann NY Acad Sci 855:349-361.

Morgan JI, Curran T (1991) Stimulus-transcription coupling in the nervous system: involvement of the inducible proto-oncogenes fos and jun. Annu Rev Neurosci 14:421-451.

Muller J, Corodimas KP, Fridel Z, LeDoux JE (1997) Functional inactivation of the lateral and basal nuclei of the amygdala by muscimol infusion prevents fear conditioning to an explicit conditioned stimulus and to contextual stimuli. Behav Neurosci 111:683-691.

Nader K, Majidishad P, Amorapanth P, LeDoux JE (2001) Damage to the lateral and central, but not other, amygdaloid nuclei prevents the acquisition of auditory fear conditioning. Learn Mem 8:156-163.

Paré D, Royer S, Smith Y, Lang EJ (2003) Contextual inhibitory gating of impulse traffic in the intra-amygdaloid network. Ann NY Acad Sci 985:78-91.

Paré D, Quirk GJ, LeDoux JE (2004) New vistas on amygdala networks in conditioned fear. J Neurophysiol 92:1-9.

Paul L, Kupferschmidt J (1975) Killing of conspecific and mouse young by male rats. J Comp Physiol Psychol 88:755-763.

Paxinos G, Watson C (1998) The rat brain in stereotaxic coordinates, Ed 4. San Diego: Academic.

Pitkänen A (2000) Connectivity of the rat amygdaloid complex. In: The amygdala, Ed 2 (Aggleton JP, ed), pp 31-115. Oxford: Oxford UP.

Power AE, McGaugh JL (2002) Cholinergic activation of the basolateral amygdala regulates unlearned freezing behavior in rats. Behav Brain Res 134:307-315.

Rosen JB (2004) The neurobiology of conditioned and unconditioned fear: a neurobehavioral system analysis of the amygdala. Behav Cogn Neurosci Rev 3:23-41.

Sah P, Faber ESL, Lopez de Armentia M, Power J (2003) The amygdaloid complex: anatomy and physiology. Physiol Rev 83:803-834.

Scalia F, Winans SS (1975) The differential projections of the olfactory bulb and accessory olfactory bulb in mammals. J Comp Neurol 161:31-56.

Shair HN, Masmela JR, Hofer MA (1999) The influence of olfaction on potentiation and inhibition of ultrasonic vocalization of rat pups. Physiol Behav 65:769-772.

Sieck MH, Baumbach HD (1974) Differential effects of peripheral and central anosmia producing techniques on spontaneous behavior patterns. Physiol Behav 13:407-425.

Singh PJ, Tucker AM, Hofer MA (1976) Effects of nasal $\mathrm{ZnSO}_{4}$ irrigation and olfactory bulbectomy on rat pups. Physiol Behav 17:373-382.

Slotnick B, Glover P, Bodyak N (2000) Does intranasal application of zinc sulfate produce anosmia in the rat? Behav Neurosci 114:814-829.

van Schaik CP, Janson CH, eds (2000) Infanticide by males and its implications. Cambridge, UK: Cambridge UP.

Vazdarjanova A, Cahill L, McGaugh JL (2001) Disrupting basolateral amygdala function impairs unconditioned freezing and avoidance in rats. Eur J Neurosci 14:709-718.

Wallace KJ, Rosen JB (2000) Predator odor as an unconditioned fear stimulus in rats: elicitation of freezing by trimethylthiazoline, a component of fox feces. Behav Neurosci 114:912-922.

Wallace KJ, Rosen JB (2001) Neurotoxic lesions of the lateral nucleus of the amygdala decrease conditioned fear but not unconditioned fear of a predator odor: comparison with electrolytic lesions. J Neurosci 21:3619-3627.

Westenbroek C, den Boer JA, ter Horst GJ (2003) Gender-specific effects of social housing on chronic stress-induced limbic Fos expression. Neuroscience 121:189-199.

Wiedenmayer CP, Barr GA (1998) Ontogeny of defensive behavior and analgesia in rat pups exposed to an adult male rat. Physiol Behav 63:261-269.

Wiedenmayer CP, Barr GA (2001a) Developmental changes in responsivity to threat are stimulus-specific in rats. Dev Psychobiol 39:1-7.

Wiedenmayer CP, Barr GA (2001b) Developmental changes in c-fos expression to an age-specific social stressor in infant rats. Behav Brain Res 126:147-157.

Wiedenmayer CP, Goodwin GA, Barr GA (2000) The effect of periaqueductal gray lesions on responses to age-specific threats in infant rats. Dev Brain Res 120:191-198.

Wiedenmayer CP, Magarinos AM, McEwen BS, Barr GA (2005) Agespecific threats induce CRF expression in the paraventricular nucleus of the hypothalamus and hippocampus of young rats. Horm Behav 47:139150. 